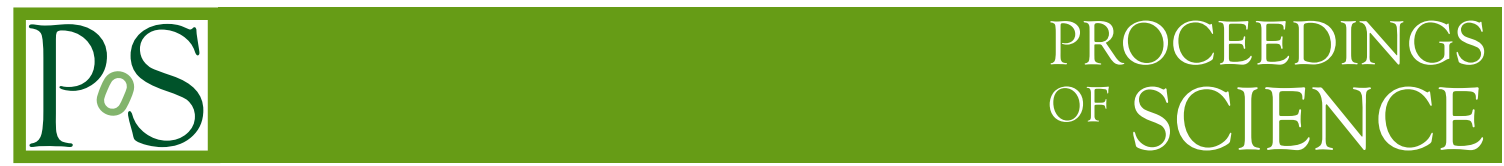

\title{
Correlation function of null polygonal Wilson loop with local operator
}

\author{
A.A. Tseytlin* \\ The Blackett Laboratory, Imperial College \\ London SW7 2AZ, U.K. \\ E-mail: atseytlinegmail.com
}

\begin{abstract}
We consider the correlator of a light-like polygonal Wilson loop with $n$ cusps with a local (dilaton or chiral primary) operator in planar $\mathscr{N}=4$ super Yang-Mills theory. Following arXiv:1107.5702 we review the structure of such correlator implied by conformal symmetry. The first non-trivial case is $n=4$ when the correlator depends on just one conformal ratio. We compute it at leading order in both the strong coupling regime (using semiclassical string theory) and the weak coupling regime (using perturbative gauge theory).
\end{abstract}

Proceedings of the Corfu Summer Institute 2011 School and Workshops on Elementary Particle Physics and Gravity

September 4-18, 2011

Corfu, Greece

${ }^{*}$ Speaker. 


\section{Introduction}

Progress in understanding duality between planar $\mathscr{N}=4$ super Yang-Mills theory and superstring theory in $A d S_{5} \times S^{5}$ based on integrability opens up the possibility of computing various observables exactly in 't Hooft coupling $\lambda$ or in string tension $\frac{\sqrt{\lambda}}{2 \pi}$. Integrability controls scaling dimensions $\Delta_{i}(\lambda)$ of primary operators $\mathscr{O}_{i}$ that determine the 2-point functions $\left\langle\mathscr{O}\left(x^{(1)}\right) \mathscr{O}\left(x^{(2)}\right)\right\rangle$ [1]. The next step is to understand 3-point functions $\left\langle\mathscr{O}_{i}\left(x^{(1)}\right) \mathscr{O}_{j}\left(x^{(2)}\right) \mathscr{O}_{k}\left(x^{(3)}\right)\right\rangle$ which, in addition to $\Delta_{i}$, are determined by non-trivial coefficient functions $C_{i j k}(\lambda)$. Higher-point correlation functions, though in principle dictated by the OPE, are much more complicated. For example, conformal invariance implies that a 4-point correlator $\left\langle\mathscr{O}_{1}\left(x^{(1)}\right) \ldots \mathscr{O}_{4}\left(x^{(4)}\right)\right\rangle$ should, in general, contain a non-trivial function of the two conformal cross-ratios $(m=0,1,2,3)$

$$
\mathrm{u}_{1}=\frac{\left|x^{(12)}\right|^{2}\left|x^{(34)}\right|^{2}}{\left|x^{(13)}\right|^{2}\left|x^{(24)}\right|^{2}}, \quad \mathrm{u}_{2}=\frac{\left|x^{(12)}\right|^{2}\left|x^{(34)}\right|^{2}}{\left|x^{(14)}\right|^{2}\left|x^{(23)}\right|^{2}}, \quad x_{m}^{(i j)} \equiv x_{m}^{(i)}-x_{m}^{(j)}
$$

and $\lambda$. Correlators of primary operators are natural observables in CFT. In addition, in a gauge theory, one may consider expectation values of Wilson loops. An important class of these, related to gluon scattering amplitudes (see [2, 3, 4] and [5] for reviews), are expectation values of Wilson loops in the fundamental representation $\left\langle W_{n}\right\rangle$ corresponding to polygons built out of null lines with $n$ cusps (located at $\left\{x_{m}^{(i)}\right\}, i=1, \ldots, n$ with $\left.\left|x^{(i, i+1)}\right|^{2}=0, x^{(n+1)} \equiv x^{(1)}\right)$. They were previously studied at weak [6] and at strong [7,2] coupling. Conformal invariance (broken by the presence of the cusps in a controllable fashion) implies [9] that for $n=4,5$ these expectation values are fixed functions of $x^{(i)}$ (depending on a few $\lambda$-dependent coefficients, in particular, on the cusp anomalous dimension [10,11]) while for $n>5$ they should depend on $3 n-15$ cross-ratios of the cusp coordinates. The first non-trivial example is $\left\langle W_{6}\right\rangle$ which is expressed in terms of a function of $\lambda$ and three cross-ratios. As suggested in [15], there is a close relation between certain correlators of local (BPS) operators and expectation values of cusped Wilson loops: a correlator

$$
K_{n}=\left\langle\hat{\mathscr{O}}\left(x^{(1)}\right) \ldots \hat{\mathscr{O}}\left(x^{(n)}\right)\right\rangle
$$

of primary operators (e.g., the highest weight part of 20' scalar) located at positions of the null cusps is proportional to the expectation value of the null polygon Wilson loop in the adjoint representation (or to $\left\langle W_{n}\right\rangle^{2}$ in the planar approximation we will consider here). More precisely,

$$
\lim _{\left|x^{(i, i+1)}\right| \rightarrow 0} K_{n} / K_{n 0}=\left\langle W_{n}\right\rangle^{2}, \quad K_{n 0} \sim \prod_{i=1}^{n}\left|x^{(i, i+1)}\right|^{-2}+\ldots
$$

is the most singular term in the tree-level $(\lambda=0)$ part of $K_{n}$.

Here we shall review the study [16] of a new observable that involves both a local operator and a cusped Wilson loop, i.e. $\left\langle W_{n} \mathscr{O}(a)\right\rangle$ where $a$ is position of the local operator. One motivation is that such correlators may lead to new simple examples where one may be able to interpolate from weak to strong coupling. In particular, in the first non-trivial case $n=4$ such correlator happens to be a function of just one non-trivial conformal ratio formed from the coordinates of the cusps $x^{(i)}$ and the operator $a_{m}$ (for $n>4$ it will be a function of $3 n-11$ conformal ratios). For comparison, in the case of a circular Wilson loop (which, in fact, may be viewed as an $n \rightarrow \infty$ limit of a regular null 
polygon) the dependence of the correlator $\left\langle W_{\infty} \mathscr{O}(a)\right\rangle$ on the location of the operator $a$ is completely fixed by conformal invariance. Determining such a function (both at weak and at strong coupling) should be easier than the function of the two conformal ratios in the 4-point correlator case or the function of the three conformal ratios in the 6-cusp Wilson loop case.

Another motivation to study such "mixed" correlators is that they may shed more light on the relation [15] between the correlators of null-separated operators and cusped Wilson loops mentioned above. That relation was verified at weak coupling, but checking it explicitly at strong coupling remains an important open problem. For example, one may start with a $(n+1)$-point correlator and consider a limit in which only $n$ of the locations of the operators become null-separated and attempt to relate this limit to $\left\langle W_{n} \mathscr{O}(a)\right\rangle$ with $a=x^{(n+1)}$.

More explicitly, since the derivative of a correlator over the gauge coupling brings down a power of the super YM action which is the same as the integrated dilaton operator, the relation

$$
\left\langle\hat{\mathscr{O}}\left(x^{(1)}\right) \ldots \hat{\mathscr{O}}\left(x^{(n)}\right)\right\rangle \sim\left\langle W_{n}\right\rangle^{2}
$$

implies that

$$
\left\langle\hat{\mathscr{O}}\left(x^{(1)}\right) \ldots \hat{\mathscr{O}}\left(x^{(n)}\right) \int d^{4} a \mathscr{O}_{d i l}(a)\right\rangle \sim 2\left\langle W_{n}\right\rangle\left\langle W_{n} \int d^{4} a \mathscr{O}_{\text {dil }}(a)\right\rangle .
$$

Assuming that the integral over $a$ can be omitted and, furthermore, the dilaton operator can be replaced by a generic local operator one may conjecture that

$$
\left\langle\hat{\mathscr{O}}\left(x^{(1)}\right) \ldots \hat{\mathscr{O}}\left(x^{(n)}\right) \mathscr{O}(a)\right\rangle \sim\left\langle W_{n}\right\rangle\left\langle W_{n} \mathscr{O}(a)\right\rangle
$$

i.e. that

$$
\lim _{\left|x^{(i)}-x^{(i+1)}\right| \rightarrow 0} \frac{\left\langle\hat{\mathscr{O}}\left(x^{(1)}\right) \ldots \hat{\mathscr{O}}\left(x^{(n)}\right) \mathscr{O}(a)\right\rangle}{\left\langle\hat{\mathscr{O}}\left(x^{(1)}\right) \ldots \hat{\mathscr{O}}\left(x^{(n)}\right)\right\rangle} \sim \frac{\left\langle W_{n} \mathscr{O}(a)\right\rangle}{\left\langle W_{n}\right\rangle}
$$

In Section 2, we shall use general symmetry considerations to determine the structure of the correlator (2.1) of a null $n$-polygon Wilson loop and a conformal primary operator. We shall explicitly discuss the case of $n=4$ where the result will be expressed in terms of a function $F$ of only one non-trivial conformal ratio (2.12) depending on the locations of the operator and the cusps. Taking the $|a| \rightarrow \infty$ limit then determines the corresponding OPE coefficient [12]. In Section 3 we shall explicitly compute the $n=4$ correlator at strong coupling using semiclassical string theory methods $[12,13]$, i.e. evaluating the vertex operator corresponding to $\mathscr{O}$ on the string surface $[7,2]$ ending on the null quadrangle. We shall explicitly determine the strong-coupling form of the function $F$ for the two cases: when $\mathscr{O}$ is the dilaton or is a chiral primary operator. In Section 4 we turn to the evaluation of this correlator at weak coupling, i.e. in perturbative gauge theory. In Section 5 we shall mention some open questions.

\section{Structure of correlation function of cusped Wilson loop and a local operator}

Below we will consider the correlation function

$$
\mathscr{C}\left(W_{n}, a\right)=\frac{\left\langle W_{n} \mathscr{O}(a)\right\rangle}{\left\langle W_{n}\right\rangle},
$$


where $W_{n}$ is a polygonal Wilson loop made out of $n$ null lines and $\mathscr{O}$ is a local scalar operator inserted at a generic point $a=\left\{a_{m}\right\}=\left(a_{0}, a_{1}, a_{2}, a_{3}\right)$. While the expectation value $\left\langle W_{n}\right\rangle$ of such Wilson loops is known to have UV divergences due to the presence of the cusps $[10,11,6]$ (enhanced in the null case) we will see that the ratio (2.1) is finite, i.e. does not require a regularization.

As follows from conformal symmetry, the non trivial part of $\left\langle W_{n}\right\rangle$ depends only on the conformally invariant ratios constructed using the coordinates of the cusps [9]. The number of such conformal ratios for $n>5$ is $4 n-n-15=3 n-15$. Here $4 n$ stands for the total number of coordinates, $n$ is the number of null conditions on the polygon lines and 15 is the dimension of the conformal group. Furthermore, we expect (2.1) to be finite, since divergences from the numerator will be canceled by divergences from the denominator. The number $3 n-15$ of independent conformal ratios is exactly the same as the one that would appear in a correlator of $n \geq 4$ primary operators located at the corners of a null polygon. In general, the structure of $n$-point correlator $\left\langle\mathscr{O}\left(x^{(1)}\right) \ldots \mathscr{O}\left(x^{(n)}\right)\right\rangle$ is fixed by conformal symmetry up to a function of conformal ratios. The number of these conformal ratios is always given by $c_{n}=4 n-\gamma_{n}$, where $4 n$ is the total number of coordinates and $\gamma_{n}$ is the number of generators of the conformal group broken by the precense of the local operators. For $n=2,3,4$ we have $\gamma_{2}=8, \gamma_{3}=12$ and $\gamma_{4}=14$ so that $c_{2}=0, c_{3}=0, c_{4}=2$. A random configuration of $n>4$ points breaks the conformal group completely, i.e. $\gamma_{n}=15$ and thus for $n>4$ we have $c_{n}=4 n-15$. If the operators are located at the corners of a null polygon we have to impose $n$ additional constraints which gives $d_{n}=3 n-\gamma_{n}$ for the number of conformal ratios, i.e. $d_{4}=-2, d_{5}=0$ and thus $d_{n}=3 n-15$ for $n>4$. Adding an operator $\mathscr{O}$ in (2.1) at a generic point brings in 4 parameters so that $\mathscr{C}\left(W_{n}, a\right)$ with $n \geq 4$ should be a non-trivial function of $3 n-11$ conformally invariant combinations $\zeta_{k}$ constructed out of the coordinates $x_{m}^{(i)}$ of the $n$ cusps and the point $a_{m}$. This is, of course, the same as the number of conformal ratios parametrising a correlator of $n+1$ operators with only $n$ points being null-separated,

$$
c_{n+1}-n=4(n+1)-15-n=3 n-11 .
$$

In general, $\mathscr{C}\left(W_{n}, a\right)$ should be a function of $n$ distances $\left|a-x^{(k)}\right|$ and $\frac{1}{2} n(n-3)$ non-zero "diagonals" of the null polygon $\left|x^{(i)}-x^{(j)}\right|, i \neq j \pm 1$. We shall use the notation: $\left|x-x^{\prime}\right|^{2}=\left(x_{m}-\right.$ $\left.x_{m}^{\prime}\right)^{2}=-\left(x_{0}-x_{0}^{\prime}\right)^{2}+\left(x_{1}-x_{1}^{\prime}\right)^{2}+\left(x_{2}-x_{2}^{\prime}\right)^{2}+\left(x_{3}-x_{3}^{\prime}\right)^{2}$. It should also transform like the operator $\mathscr{O}(a)$ with dimension $\Delta$ under (i) dilatations and (ii) inversions, i.e. (i) $\mathscr{C} \rightarrow h^{-\Delta} \mathscr{C}$ under $x^{(i)} \rightarrow h x^{(i)}, a \rightarrow h a$, and (ii) $\mathscr{C} \rightarrow|a|^{2 \Delta} \mathscr{C}$ under $a_{m} \rightarrow \frac{a_{m}}{\mid x x^{2}}, x_{m}^{(i)} \rightarrow \frac{x_{m}^{(i)}}{\left|x^{(i)}\right|^{2}}$. The large $|a|$ behavior of $\mathscr{C}$ can be fixed by consistency with the expected OPE expansion: for small Wilson loop one may represent it in terms of a sum of local operators [12]

$$
\frac{W_{n}}{\left\langle W_{n}\right\rangle}=1+\sum_{k} c_{k} \mathrm{r}^{\Delta_{k}} \mathscr{O}_{k}(0)+\ldots
$$

where $\mathrm{r}$ is the characteristic size of a loop, $\mathscr{O}_{k}$ are conformal primary operators with dimensions $\Delta_{k}$, and dots stand for contributions of their conformal descendants. Taking the position $a$ of the operator $\mathscr{O}$ to be far away from the null polygon one should then get

$$
\left.\frac{\left\langle W_{n} \mathscr{O}(a)\right\rangle}{\left\langle W_{n}\right\rangle}\right|_{|a| \rightarrow \infty} \sim\left\langle\mathscr{O}^{\dagger}(0) \mathscr{O}(a)\right\rangle \sim \frac{1}{|a|^{2 \Delta}},
$$


where $\mathscr{O}^{\dagger}$ conjugate to $\mathscr{O}$ is among the operators present in (2.3). Since all distances $\left|a-x^{(k)}\right|$ between the operator and the cusps should appear on an equal footing this suggests the following ansatz

$$
\mathscr{C}\left(W_{n}, a\right)=\frac{\mathscr{F}\left(a, x^{(i)}\right)}{\prod_{k=1}^{n}\left|a-x^{(k)}\right|^{\frac{2}{n} \Delta}},
$$

where $\mathscr{F}$ is finite in the $|a| \rightarrow \infty$ limit, i.e. it may depend on $\left|a-x^{(k)}\right|$ only through their ratios. The dependence of $\mathscr{F}$ on $\left|x^{(i)}-x^{(j)}\right|$ is constrained by the transformations under dilatations and inversions mentioned above which implies that under these two transformations we should have

$$
\text { (i) } \mathscr{F} \rightarrow h^{\Delta} \mathscr{F}, \quad(\text { ii }) \mathscr{F} \rightarrow\left(\left|x^{(1)}\right| \ldots\left|x^{(n)}\right|\right)^{-\frac{2}{n} \Delta} \mathscr{F} .
$$

We are led to the following expression for (2.1)

$$
\mathscr{C}\left(W_{n}, a\right)=\frac{\prod_{i<j-1}^{n}\left|x^{(i)}-x^{(j)}\right|^{\frac{2}{n(n-3)} \Delta}}{\prod_{k=1}^{n}\left|a-x^{(k)}\right|^{\frac{2}{n} \Delta}} F\left(\zeta_{1}, \ldots, \zeta_{3 n-11}\right) .
$$

In general, $\Delta$ and $F$ in (2.7) may depend also on the coupling $\lambda$, i.e. they may look different at weak and at strong coupling, but the general structure (2.7) should be universal.

The same structure (2.7) follows also from the general form of the correlator of local operators if the relation (1.7) is assumed to be true. As is well know, conformal invariance implies that a correlator of $q$ primary operators $\mathscr{O}_{i}\left(x^{(i)}\right)$ of dimensions $\Delta_{i}$ at generic positions should be

$$
\begin{aligned}
& \left\langle\mathscr{O}_{1}\left(x^{(1)}\right) \ldots \mathscr{O}_{q}\left(x^{(q)}\right)\right\rangle=T_{q} \mathrm{~F}_{q}\left(\mathrm{u}_{1}, \ldots, \mathrm{u}_{c_{q}}\right), \quad T_{q} \equiv \prod_{i<j}^{q}\left|x^{(i)}-x^{(j)}\right|^{-\gamma_{i j}}, \\
& \gamma_{i j}=\frac{2}{q-2}\left(\Delta_{i}+\Delta_{j}-\frac{1}{q-1} \sum_{k=1}^{q} \Delta_{k}\right), \quad c_{4}=2, \quad c_{q>4}=4 q-15,
\end{aligned}
$$

where $\mathrm{F}_{q}$ is a function of conformally-invariant cross-ratios. Then Considering $q=n+1$ with $n$ operators being the same, $\mathscr{O}_{k}=\hat{\mathscr{O}}, \Delta_{k}=\hat{\Delta}$ and $\mathscr{O}_{n+1}=\mathscr{O}, \Delta_{n+1}=\Delta$ we find

$$
\frac{T_{n+1}}{T_{n}}=\frac{\prod_{i<j}^{n}\left|x^{(i)}-x^{(j)}\right|^{\frac{2}{n(n-1)} \Delta}}{\prod_{k=1}^{n}\left|a-x^{(k)}\right|^{\frac{2}{n} \Delta}}, \quad a \equiv x^{(n+1)} .
$$

To get a non-trivial expression in the null-separation limit $\left|x^{(i)}-x^{(i+1)}\right| \rightarrow 0$ we will need to assume that $n$ of such vanishing factors in numerator of (2.10) get cancelled against similar factors in some cross-ratios contained in $\mathrm{F}_{n+1} / \mathrm{F}_{n}$. That will change the powers of the remaining $\frac{1}{2} n(n-1)-n=$ $\frac{1}{2} n(n-3)$ non-zero factors $\left|x^{(i)}-x^{(j)}\right|$ in (2.10) and also reduce the total number of non-trivial conformal ratios (now denoted by $\zeta_{r}$ ) by $n$ as in (2.2). The result will then have the same form as in (2.7). Indeed, the combination one needs to multiply (2.10) by to cancel the vanishing $\left|x^{(i)}-x^{(i+1)}\right|$ factors in the numerator and to match the prefactor in (2.7) with $\mu_{i j}=\frac{2 \Delta}{n(n-3)}$ is $\left(x^{(n+1)} \equiv x^{(1)}\right)$

$$
\frac{\prod_{i<j-1}^{n}\left|x^{(i)}-x^{(j)}\right|^{\frac{4}{n(n-1)(n-3)} \Delta}}{\prod_{k=1}^{n}\left|x^{(k)}-x^{(k+1)}\right|^{\frac{2}{n(n-1)}} \Delta} .
$$


Let us now look in detail at the first non-trivial example: $n=4{ }^{1}$ Here the number of variables $\zeta_{k}$ is $3 \times 4-11=1$, i.e. $F$ should be a function of a single variable $\zeta_{1} \equiv \zeta$. For $n=5$ the number of conformal ratios is already 4 . This makes the correlation function (2.1) for $n=4$ a particularly interesting and simple case to study.

As it follows from the above discussion, this variable $\zeta$ can be viewed as the unique conformal ratio which one can build out of the coordinates $x_{m}^{(i)}(i=1, \ldots, 4)$ of 4 cusps and the location $a_{m}$ of the operator $\mathscr{O}$. Assuming that the null quadrangle is ordered as $x^{(1)}, x^{(2)}, x^{(3)}, x^{(4)}$ (i.e. $\mid x^{(1)}-$ $\left.\left.x^{(2)}\right|^{2}=\left|x^{(2)}-x^{(3)}\right|^{2}=\left|x^{(3)}-x^{(4)}\right|^{2}=\left|x^{(4)}-x^{(1)}\right|^{2}=0\right)$ it is easy to see that the unique non-trivial conformally-invariant combination of these 5 points is

$$
\zeta=\frac{\left|a-x^{(2)}\right|^{2}\left|a-x^{(4)}\right|^{2}\left|x^{(1)}-x^{(3)}\right|^{2}}{\left|a-x^{(1)}\right|^{2}\left|a-x^{(3)}\right|^{2}\left|x^{(2)}-x^{(4)}\right|^{2}} .
$$

In this case there is also a unique choice for the $x^{(i)}$-dependent factor in (2.7): $\left(\mid x^{(1)}-x^{(3)} \| x^{(2)}-\right.$ $\left.x^{(4)} \mid\right)^{\Delta / 2}$ that ensures the right dimensionality of the result. We conclude that the correlation function (2.1) for $n=4$ should have the following general form

$$
\mathscr{C}\left(W_{4}, a\right)=\frac{\left(\left|x^{(1)}-x^{(3)}\right|\left|x^{(2)}-x^{(4)}\right|\right)^{\Delta / 2}}{\prod_{i=1}^{4}\left|a-x^{(i)}\right|^{\Delta / 2}} F(\zeta),
$$

where $\Delta$ is the dimension of the operator $\mathscr{O}$ and $\zeta$ is given by (2.12).

As discussed above, the same conclusion applies also to a correlator of 4 equivalent nullseparated operators and an extra operator $\mathscr{O}$. Indeed, for $n=4$ it is easy to see that (2.10) is to be multiplied, according to (2.11), by

$$
\frac{\left(\left|x^{(1)}-x^{(3)}\right|\left|x^{(2)}-x^{(4)}\right|\right)^{\Delta / 3}}{\prod_{k=1}^{4}\left|x^{(k)}-x^{(k+1)}\right|^{\Delta / 6}},
$$

which is a product of two cross-ratios in power $\Delta / 6$.

It is interesting to note that depending on just one conformal ratio, the $n=4$ correlator (2.13) is an "intermediate" case between a 3-point function $\left\langle\mathscr{O}\left(x^{(1)}\right) \mathscr{O}\left(x^{(2)}\right) \mathscr{O}\left(x^{(3)}\right)\right\rangle$ which is completely fixed by conformal invariance (up to a function of the coupling) and a generic 4-point function $\left\langle\mathscr{O}\left(x^{(1)}\right) \ldots \mathscr{O}\left(x^{(4)}\right)\right\rangle$ which depends on two conformal ratios.

In the limit when $|a| \rightarrow \infty$ we get

$$
\begin{aligned}
& \mathscr{C}\left(W_{4}, a\right)_{|a| \rightarrow \infty}=\frac{\mathrm{C}}{|a|^{2 \Delta}}, \\
& \mathrm{C} \equiv\left(\left|x^{(1)}-x^{(3)}\right|\left|x^{(2)}-x^{(4)}\right|\right)^{\Delta / 2} F\left(\zeta_{\infty}\right), \quad \zeta_{\infty}=\frac{\left|x^{(1)}-x^{(3)}\right|^{2}}{\left|x^{(2)}-x^{(4)}\right|^{2}},
\end{aligned}
$$

where $\mathrm{C}$ thus determines the corresponding OPE coefficient in (2.3).

Another special limit is when the position of the operator approaches the location of one of the cusps, e.g., $a \rightarrow x^{(1)}$. Setting $a_{m}=x_{m}^{(1)}+\varepsilon \alpha_{m}, \varepsilon \rightarrow 0$, and using that the vectors $x^{(1)}-x^{(2)}$ and $x^{(1)}-x^{(4)}$ are null we find from (2.12) that $\zeta$ is, generically, finite in this limit and is given by

$$
\zeta_{a \rightarrow x^{(1)}}=\frac{4 \alpha \cdot\left(x^{(1)}-x^{(2)}\right) \alpha \cdot\left(x^{(1)}-x^{(4)}\right)}{\alpha^{2}\left|x^{(2)}-x^{(4)}\right|^{2}}, \quad a_{m}=x_{m}^{(1)}+\varepsilon \alpha_{m} .
$$

\footnotetext{
${ }^{1}$ The case of $n=3$ is trivial as there is no solution for coordinates of a null triangle in real $4 \mathrm{~d}$ Minkowski space.
} 
Similarly, the limit of the pre-factor in (2.13) is

$$
\prod_{i=1}^{4}\left|a-x^{(i)}\right|_{a \rightarrow x^{(1)}}^{\Delta / 2} \rightarrow 4 \varepsilon^{\Delta} \alpha \cdot\left(x^{(1)}-x^{(2)}\right) \alpha \cdot\left(x^{(1)}-x^{(4)}\right)\left|x^{(1)}-x^{(3)}\right|^{2} .
$$

Thus

$$
\mathscr{C}\left(W_{4}^{(r e g)}, a\right)_{a \rightarrow x^{(1)}} \sim \frac{1}{\left|a-x^{(1)}\right|^{\Delta}} .
$$

Note that this is the same behavior that would be expected if the Wilson loop were replaced by a product of 4 same-type operators (e.g., scalar operators as in [15]) at the positions of the cusps: $\left\langle W_{4} \mathscr{O}(a)\right\rangle \rightarrow\left\langle\hat{\mathscr{O}}\left(x^{(1)}\right) \ldots \hat{\mathscr{O}}\left(x^{(4)}\right) \mathscr{O}(a)\right\rangle$. Then the limit $a \rightarrow x^{(1)}$ would be determined by the OPE, $\hat{\mathscr{O}}\left(x^{(1)}\right) \mathscr{O}(a) \sim \frac{1}{\left|a-x^{(1)}\right|^{\Delta}} \hat{\mathscr{O}}\left(x^{(1)}\right)$. One can explicitly verify [16] the general form (2.7), (2.13) of the correlator (2.1) at leading orders in the strong-coupling (section 3) and the weak-coupling (section 6) expansions and compute the corresponding function $F$.

\section{Correlation function of 4-cusp Wilson loop with a local operator at strong coupling}

Here we will compute (2.1) for $n=4$ corresponding to the 4-cusp Wilson loop at strong coupling. The result will have the expected form (2.13) and we will explicitly determine the function $F(\zeta)$. We shall always consider the planar limit of maximally supersymmetric Yang-Mills theory and assume that the operator $\mathscr{O}$ is such that for large "t Hooft coupling $\lambda$ its dimension $\Delta$ is much smaller than $\sqrt{\lambda}$. In particular, $\mathscr{O}$ will be chosen as the dilaton operator or the chiral primary operator. We shall follow the same semiclassical string theory approach that was used in the case of the circular Wilson loop in $[12,13]$ (see also [23, 24, 22, 26, 14]). In string-theory description the local operator $\mathscr{O}(a)$ is represented by a marginal vertex operator [27]

$$
\mathrm{V}(a)=\int d^{2} \xi V[X(\xi) ; a]
$$

where $X$ stands for the $2 \mathrm{~d}$ fields that enter the $A d S_{5} \times S^{5}$ superstring action. In general, (2.1) is then given by

$$
\mathscr{C}\left(W_{n}, a\right)=\frac{1}{\left\langle W_{n}\right\rangle} \int[d X] \mathrm{V}(a) e^{-I[X]}
$$

Here $I$ is the string action proportional to the tension $T=\frac{\sqrt{\lambda}}{2 \pi}$ and the path integral is performed over the euclidean world-sheets with topology of a disc (we consider only the planar approximation) and the boundary conditions set out by the Wilson loop at $z=0$. Considering the limit when $\sqrt{\lambda} \gg 1$ and assuming that the operator represented by $\mathrm{V}$ is "light" [22] (i.e. the corresponding scaling dimension and charges are much smaller than $\sqrt{\lambda}$ ) one concludes that this path integral is dominated by the same semiclassical string surface as in the absence of V, i.e. as in the case of $\left\langle W_{n}\right\rangle$. The resulting leading-order value of (3.2) is then given by (3.1) evaluated on this classical solution, i.e.

$$
\mathscr{C}\left(W_{n}, a\right)_{\sqrt{\lambda} \gg 1}=\left(\int d^{2} \xi V[X(\xi) ; a]\right)_{\text {semicl. }} .
$$


One simple case is when the local operator $\mathscr{O}$ is the dilaton operator $\mathscr{O}_{\text {dil }} \sim \operatorname{tr}\left(F_{m n}^{2} Z^{j}\right)+\ldots$ (where we included also the R-charge $j$ dependence). The corresponding vertex operator has the form [22]

$$
\begin{aligned}
& \mathrm{V}_{d i l}(a)=c_{d i l} \int d^{2} \xi\left[\frac{z}{z^{2}+\left(x_{m}-a_{m}\right)^{2}}\right]^{\Delta} \mathrm{X}^{j} U_{d i l}, \\
& \mathrm{X}^{j}=\left(\cos \theta e^{i \varphi}\right)^{j}, \quad \Delta=4+j,
\end{aligned}
$$

where $j \ll \sqrt{\lambda}$ is an angular momentum along $S^{1}$ in $S^{5}$. The operator $U_{d i l}$ equals the $A d S_{5} \times S^{5}$ Lagrangian

$$
U_{d i l}=\mathscr{L}=\mathscr{L}_{A d S_{5}}+\mathscr{L}_{S^{5}}+\text { fermions }, \quad \mathscr{L}_{A d S_{5}}=\frac{1}{z^{2}}\left[\left(\partial_{\alpha} z\right)^{2}+\left(\partial_{\alpha} x_{m}\right)^{2}\right] .
$$

Furthermore, $c_{d i l}$ is the normalization coefficient given by [12, 23, 22]

$$
c_{d i l}=\frac{\sqrt{\lambda}}{8 \pi N} \sqrt{(j+1)(j+2)(j+3)} .
$$

In the case of $j=0$

$$
j=0: \quad \Delta=4, \quad c_{d i l}=\frac{\sqrt{6} \sqrt{\lambda}}{8 \pi N} .
$$

Let us start with the case when the Wilson loop is the regular (i.e. equal-sided) quadrangle with 4 cusps. The classical euclidean world-sheet surface in $A d S_{5}$ ending on this Wilson loop was found in [2] and is given by ${ }^{2}$

$$
\begin{aligned}
& z=\frac{\mathrm{r}}{\cosh u \cosh v}, \quad x_{0}=\mathrm{r} \tanh u \tanh v, \\
& x_{1}=\mathrm{r} \tanh u, \quad x_{2}=\mathrm{r} \tanh v, \quad x_{3}=0 ; \quad u, v \in(-\infty, \infty) .
\end{aligned}
$$

Here $z$ is the radial direction of the Poincare patch of $A d S_{5}$ and $x_{m}=\left(x_{0}, x_{1}, x_{2}, x_{3}\right)$ are the coordinates on the boundary. The parameter $r$ corresponds to the overall scale of the loop. To simplify later formulas we will set $\mathrm{r}=1$ (it is easy to restore $\mathrm{r}$ by simply replacing $z \rightarrow \mathrm{r}^{-1} z, x_{m} \rightarrow \mathrm{r}^{-1} x_{m}$ ). The cusps correspond to $(u, v) \rightarrow( \pm \infty, \pm \infty)$ and thus are located at

$$
\begin{array}{ll}
x^{(1)}=(1,1,1,0), & x^{(2)}=(-1,1,-1,0), \\
x^{(3)}=(1,-1,-1,0), & x^{(4)}=(-1,-1,1,0),
\end{array}
$$

Substituting (3.10) into (2.12) gives the following explicit form of the conformal ratio $\zeta$ that is expected to appear in the correlator

$$
\begin{aligned}
& \zeta=\frac{\left(\frac{1}{2} \mathrm{q}-a_{0}-a_{1}+a_{2}\right)\left(\frac{1}{2} \mathrm{q}-a_{0}+a_{1}-a_{2}\right)}{\left(\frac{1}{2} \mathrm{q}+a_{0}-a_{1}-a_{2}\right)\left(\frac{1}{2} \mathrm{q}+a_{0}+a_{1}+a_{2}\right)} \\
& \mathrm{q} \equiv 1-a_{0}^{2}+a_{1}^{2}+a_{2}^{2}+a_{3}^{2} .
\end{aligned}
$$

Substituting the classical solution (3.9) into (3.4) we obtain ${ }^{3}$

$$
\mathscr{C}_{d i l}\left(W_{4}^{(r e g)}, a\right)=2 c_{d i l} \int_{-\infty}^{\infty} d u d v\left[\frac{(\cosh u \cosh v)^{-1}}{\mathrm{q}-2 a_{1} \tanh u-2 a_{2} \tanh v+2 a_{0} \tanh u \tanh v}\right]^{4},
$$

\footnotetext{
${ }^{2}$ Here $(u, v)$ cover the full plane, but since infinity is not identified the world sheet has topology of a disc.

${ }^{3}$ Below in this section the expression for a correlator will always stand for its leading $\sqrt{\lambda} \gg 1$ value.
} 
where $\mathrm{q}$ is given by (3.12) and we used the fact that on the solution (3.9) one has $U_{\text {dil }}=2$ (note also that here $\int d^{2} \xi=\int d u d v$ ). The integral is straightforward to do and we get

$$
\mathscr{C}_{d i l}\left(W_{4}^{(r e g)}, a\right)=c_{d i l} \frac{16 a_{1} a_{2}-8 \mathrm{q} a_{0}-\left(\mathrm{q}^{2}+4 a_{0}^{2}-4 a_{1}^{2}-4 a_{2}^{2}\right) \log \zeta}{12\left(\mathrm{q} a_{0}-2 a_{1} a_{2}\right)^{3}} .
$$

where we have used (3.11). The result is thus finite, in contrast to the area of the 4-cusp surface that requires a regularization [2]. The result (3.14) is indeed consistent with eq. (2.13) for $\Delta=4$ with

$$
F(\zeta)=\frac{c_{d i l}}{3} \frac{\zeta}{(\zeta-1)^{3}}[-2(\zeta-1)+(\zeta+1) \log \zeta]
$$

In the limit $|a| \rightarrow \infty$ (see (2.15)) we get $\zeta_{\infty}=1$ and thus

$$
\mathscr{C}_{\text {dil }}\left(W_{4}^{(r e g)}, a\right)_{|a| \rightarrow \infty}=\frac{32 c_{d i l} \mathrm{r}^{4}}{9|a|^{8}} .
$$

which determines the OPE coefficient of $\mathscr{O}_{\text {dil }}$ in the expansion (2.3) of the Wilson loop $W_{4}^{(r e g)}{ }^{4}$

In the limit when $a$ approaches a cusp $\left(a_{m}=x_{m}^{(1)}+\varepsilon \alpha_{m}, \varepsilon \rightarrow 0\right)$ (see (2.17),(2.18)) we get

$$
\begin{aligned}
& \mathscr{C}_{d i l}\left(W_{4}^{(r e g)}, a\right)_{a \rightarrow x^{(1)}} \rightarrow-\frac{2}{3 \varepsilon^{4}} \frac{1}{\left[-3 \alpha_{0}^{2}+\alpha_{3}^{2}+\left(\alpha_{1}-\alpha_{2}\right)^{2}+2 \alpha_{0}\left(\alpha_{1}+\alpha_{2}\right)\right]^{2}} \\
& \times\left[1-\frac{\alpha_{3}^{2}+\left(\alpha_{1}+\alpha_{2}-\alpha_{0}\right)^{2}}{-3 \alpha_{0}^{2}+\alpha_{3}^{2}+\left(\alpha_{1}-\alpha_{2}\right)^{2}+2 \alpha_{0}\left(\alpha_{1}+\alpha_{2}\right)} \log \frac{\alpha_{1}^{2}+\alpha_{2}^{2}+\alpha_{3}^{2}-\alpha_{0}^{2}}{2\left(\alpha_{0}-\alpha_{1}\right)\left(\alpha_{0}-\alpha_{2}\right)}\right] .
\end{aligned}
$$

The behavior $\varepsilon^{-\Delta}=\varepsilon^{-4}$ is in agreement with the general expression (2.19).

The above calculation can be generalized to the case of an irregular quadrangle, i.e. the one with unequal diagonals $s \neq t$. The corresponding solution can be found by applying a conformal transformation to (3.9) [2]

$$
\begin{aligned}
& z=f(u, v) \cosh u \cosh v, \quad x_{0}=\sqrt{1+b^{2}} f(u, v) \tanh u \tanh v \\
& x_{1}=f(u, v) \tanh u, \quad x_{2}=f(u, v) \tanh v, \quad x_{3}=0 \\
& f(u, v) \equiv \frac{\mathrm{r}}{1+b \tanh u \tanh v}, \quad|b| \leq 1
\end{aligned}
$$

$b=0$ corresponds to the regular quadrangle case (3.9). The cusps are found by taking $(u, v) \rightarrow$ $( \pm \infty, \pm \infty)$ and are located at (cf. (3.10); here we set $r=1)$

$$
\begin{aligned}
& x_{m}^{(1)}=\left(\frac{\sqrt{1+b^{2}}}{1+b}, \frac{1}{1+b}, \frac{1}{1+b}, 0\right), \quad x_{m}^{(2)}=\left(-\frac{\sqrt{1+b^{2}}}{1-b}, \frac{1}{1-b}, \frac{-1}{1-b}, 0\right), \\
& x_{m}^{(3)}=\left(\frac{\sqrt{1+b^{2}}}{1+b}, \frac{-1}{1+b}, \frac{-1}{1+b}, 0\right), \quad x_{m}^{(4)}=\left(-\frac{\sqrt{1+b^{2}}}{1-b}, \frac{-1}{1-b}, \frac{1}{1-b}, 0\right) .
\end{aligned}
$$

The Wilson loop is the quadrangle $x^{(1)}, x^{(2)}, x^{(3)}, x^{(4)}$. After the same steps as in the case of the regular quadrangle we find that the resulting expression for the Wilson loop can indeed be written

\footnotetext{
${ }^{4}$ The same expression can be obtained by taking $|a|$ large directly in (3.4) and doing the resulting simple integral $\sim|a|^{-8} \int d^{2} \xi z^{4}$.
} 
as (2.13), where $\Delta=4, x^{(i)}$ 's are given by (3.19) and

$$
\begin{aligned}
& \left|x^{(1)}-x^{(3)}\right|^{2}\left|x^{(2)}-x^{(4)}\right|^{2}=\frac{64 \mathrm{r}^{4}}{\left(1-b^{2}\right)^{2}}, \\
& F(\zeta)=\frac{c_{d i l}}{3} \frac{\zeta}{(\zeta-1)^{3}}[-2(\zeta-1)+(\zeta+1) \log \zeta] .
\end{aligned}
$$

The function $F(\zeta)$ is thus the same as in (3.15), as expected.

The above discussion can be generalized also to the case when the dilaton operator (3.4) carries an angular momentum $j$ along $S^{1} \subset S^{5}$. Here

$$
\mathscr{C}_{\text {dil }}\left(W_{4}^{(\text {irreg })}, a_{0}\right)=\frac{2 \pi c_{\text {dil }}}{\left(1-a_{0}^{2}\right)^{\Delta}}\left(\frac{\Gamma\left[\frac{\Delta}{2}\right]}{\Gamma\left[\frac{\Delta+1}{2}\right]}\right)^{2}{ }_{2} F_{1}\left(\frac{1}{2}, \frac{\Delta}{2}, \frac{\Delta+1}{2}, \rho^{2}\right),
$$

where ${ }_{2} F_{1}$ is the hypergeometric function and $\rho$ is a function of $a_{0}$ given by

$$
\rho \equiv \frac{2 \tilde{a}_{0}}{1-a_{0}^{2}}=\frac{2 a_{0} \sqrt{1+b^{2}}-b\left(1+a_{0}^{2}\right)}{1-a_{0}^{2}} .
$$

To extract $F$ in (2.13) we have to multiply (3.22) by the factor

$$
\left|x^{(1)}-x^{(3)}\right|^{-\Delta / 2}\left|x^{(2)}-x^{(4)}\right|^{-\Delta / 2} \prod_{i=1}^{4}\left|x_{m}^{(i)}-a_{m}\right|^{\Delta / 2} .
$$

This gives

$$
F(\zeta(\rho))=2^{-\frac{3}{2} \Delta+1} \pi c_{d i l}\left(\frac{\Gamma\left[\frac{\Delta}{2}\right]}{\Gamma\left[\frac{\Delta+1}{2}\right]}\right)^{2}\left(1-\rho^{2}\right)^{\Delta / 2}{ }_{2} F_{1}\left(\frac{1}{2}, \frac{\Delta}{2}, \frac{\Delta+1}{2}, \rho^{2}\right) .
$$

Finally, we can express $\rho$ in terms of $\zeta$

$$
\rho=\frac{1-\sqrt{\zeta}}{1+\sqrt{\zeta}}
$$

One can check that setting $j=0$, i.e. $\Delta=4$, gives back our earlier expression (3.21).

A similar computation can be done with the chiral primary operator $\mathscr{O}_{j}=\operatorname{tr} Z^{j}$ instead of the dilaton operator. The bosonic part of the corresponding vertex operator [12, 23, 22] can be written in a form similar to (3.4)

$$
\begin{aligned}
& \mathrm{V}_{j}(a)=c_{j} \int d^{2} \xi\left[\frac{z}{z^{2}+\left(x_{m}-a_{m}\right)^{2}}\right]^{\Delta} \mathrm{X}^{j} U, \\
& \Delta=j, \quad c_{j}=\frac{\sqrt{\lambda}}{8 \pi N} \sqrt{j}(j+1),
\end{aligned}
$$

where $\mathrm{X}^{j}$ is the same as in (3.4) while the 2-derivative $U$ part is more complicated [26]

$$
\begin{aligned}
& U=U_{1}+U_{2}+U_{2}, \quad U_{1}=\frac{1}{z^{2}}\left[\left(\partial_{\alpha} x_{m}\right)^{2}-\left(\partial_{\alpha} z\right)^{2}\right]-\mathscr{L}_{S^{5}}, \\
& U_{2}=\frac{8}{\left(z^{2}+|x-a|^{2}\right)^{2}}\left[|x-a|^{2}\left(\partial_{\alpha} z\right)^{2}-\left[\left(x_{m}-a_{m}\right) \partial_{\alpha} x_{m}\right]^{2}\right], \\
& U_{3}=\frac{8\left(|x-a|^{2}-z^{2}\right)}{z\left(z^{2}+|x-a|^{2}\right)^{2}}\left(x_{n}-a_{n}\right) \partial_{\alpha} x_{n} \partial_{\alpha} z .
\end{aligned}
$$


For simplicity, we will consider the case of the regular 4-cusp Wilson loop; the corresponding solution (3.9) does not deepend on $S^{5}$ coordinates so $\mathrm{X}^{j}=1$.

To find the function $F(\zeta)$ in (2.13) it is sufficient, as in section 3.1.3, to choose the special case of $a=\left(a_{0}, 0,0,0\right)$. Then

$$
\begin{aligned}
\mathscr{C}_{j}\left(W_{4}^{(r e g)}, a_{0}\right)=\frac{2 c_{j}}{\left(1-a_{0}^{2}\right)^{j}} \int_{-\infty}^{\infty} d u d v\left[\frac{(\cosh u \cosh v)^{-1}}{1+\rho \tanh u \tanh v}\right]^{j+2} \\
\times\left[1+\rho^{2}-(\sinh u \sinh v+\rho \cosh u \cosh v)^{2}\right]
\end{aligned}
$$

For an arbitrary $j$ this integral is rather complicated but can be easily done for specific values of $j$. For instance, for $j=2$ we obtain:

$$
\mathscr{C}_{2}\left(W_{4}^{(r e g)}, a_{0}\right)=\frac{4 c_{2}}{3\left(1-a_{0}^{2}\right)^{2} \rho} \log \frac{\rho+1}{\rho-1} .
$$

As a result, we find (cf. (3.15))

$$
j=2: \quad F(\zeta)=\frac{c_{2}}{3} \frac{\sqrt{\zeta}}{\zeta-1} \log \zeta
$$

\section{Correlation function of cusped Wilson loop with dilaton operator at weak coupling}

Let us now consider the computation of the correlator (2.1)

$$
\mathscr{C}\left(W_{n}, a\right)=\frac{\left\langle W_{n} \mathscr{O}(a)\right\rangle}{\left\langle W_{n}\right\rangle},
$$

in the weakly coupled planar $S U(N) \mathscr{N}=4$ supersymmetric gauge theory. Here the expectation values are computed using gauge theory path integral and ${ }^{5}$

$$
W_{n}=\frac{1}{N} \operatorname{tr} \mathscr{P} e^{i g \oint_{\gamma} A_{m} d x^{m}}
$$

Here we rescaled the fields with the coupling constant $g$ (with $\lambda=g^{2} N$ ) so that the $\mathscr{N}=4$ Lagrangian is

$$
\mathscr{L}_{\mathscr{N}=4}=-\frac{1}{4} \operatorname{tr}\left(F_{m n}^{2}+\ldots\right)
$$

with $g$ appearing only in the vertices. We use the conventions

$$
A_{m}=A_{m}^{r} T^{r}, \quad \operatorname{tr}\left(T^{r} T^{s}\right)=\delta^{r s}, \quad r, s=1, \ldots, N^{2}-1 .
$$

The path $\gamma$ in (4.2) is the union of $n$ null segments of the form

$$
\gamma_{m}^{(i)}(t)=x_{m}^{(i)}+t\left(x_{m}^{(i+1)}-x_{m}^{(i)}\right), \quad t \in[0,1],
$$

\footnotetext{
${ }^{5}$ The additional coupling to the scalars in the locally-supersymmetric Wilson loop [33] drops out because the null polygon contour consists of null lines.
} 
where $x_{m}^{(i)}(i=1, \ldots, n)$ denote the locations of the cusps. The dilaton operator (which is a supersymmetry descendant of $\operatorname{tr} Z^{2}$ ) is essentially the $\mathscr{N}=4$ gauge theory Lagrangian up to a total derivative (see, e.g., [34] $)^{6}$

$$
\mathscr{O}_{d i l}=\hat{c}_{d i l} \operatorname{tr}\left(F_{m n}^{2}+\Phi^{I} \partial^{2} \Phi^{I}+\bar{\psi} \gamma \cdot \partial \psi+\ldots\right),
$$

where $\Phi^{I}$ are the scalars and $\psi$ are the fermions and we did not write explicitly the terms of order $g$ and $g^{2}$. The normalization coefficient $\hat{c}_{d i l}$ is given by [35]

$$
\hat{c}_{d i l}=\frac{\pi^{2}}{4 \sqrt{3} N} .
$$

The leading order contribution to (4.1) (to which we will refer as the "tree level" one) is proportional to $g^{2}$ as one can easily see from (4.1), (4.2). To compute (4.1) to this order we have to expand $W_{n}$ to order $g^{2}$. Hence, we can set $g=0$ in the Lagrangian (4.3) and in the dilaton operator (4.6). Therefore, for the purpose of computing the leading order term in (4.1) we can take

$$
\mathscr{O}_{d i l} \rightarrow \hat{c}_{d i l} \operatorname{tr} F_{m n}^{2}=2 \hat{c}_{d i l}\left(\partial_{m} A_{n}^{r} \partial^{m} A^{n r}-\partial_{m} A_{n}^{r} \partial^{n} A^{m r}\right) .
$$

The gluon propagator in the above conventions is

$$
\left\langle A_{m}^{r}(x) A_{n}^{s}(0)\right\rangle=-\frac{1}{4 \pi^{2}|x|^{2}} \eta_{m n} \delta^{r s} .
$$

We will see that just like at strong coupling, the weak coupling correlator (4.1) is finite, i.e. we do not need to introduce a UV regularization in (4.9). Also note that to compute (4.1) to order $g^{2}$ we can replace $\left\langle W_{n}\right\rangle$ in the denominator with unity. Therefore, we obtain

$$
\begin{aligned}
& \mathscr{C}_{\text {dil }}^{\left(g^{2}\right)}\left(W_{n}, a\right)=\left\langle W_{n} \mathscr{O}_{\text {dil }}(a)\right\rangle_{\text {tree }} \\
& =-\frac{2 \hat{c}_{d i l} g^{2}}{N}\left\langle\mathscr{P} \oint A_{k}^{s}(x) d x^{k} \oint A_{l}^{s}\left(x^{\prime}\right) d x^{\prime l}\left(\partial_{p} A_{q}^{r} \partial^{p} A^{q r}-\partial_{p} A_{q}^{r} \partial^{q} A^{p r}\right)(a)\right\rangle .
\end{aligned}
$$

The path ordering symbol $\mathscr{P}$ means that $x^{\prime}$ in the second integral is placed between the origin (an arbitrary point along the loop, for instance one of the cusps) and $x$. Now using that

$$
\left\langle A_{k}^{r}(x) \partial_{p} A_{q}^{s}(a)\right\rangle=-\frac{1}{4 \pi^{2}} \frac{\partial}{\partial a^{p}} \frac{\eta_{k q} \delta^{r s}}{|a-x|^{2}}=-\frac{1}{2 \pi^{2}} \frac{(a-x)_{p} \eta_{k q} \delta^{r s}}{|a-x|^{4}},
$$

and performing the Wick contractions we obtain $\left(\lambda=g^{2} N\right)$

$$
\begin{aligned}
\mathscr{C}_{d i l}^{\left(g^{2}\right)}\left(W_{n}, a\right)=-\frac{\hat{c}_{d i l} \lambda}{\pi^{4}} & \mathscr{P} \\
& \left(\oint \oint \left[\frac{(a-x) \cdot\left(x-x^{\prime}\right)}{|a-x|^{4}\left|a-x^{\prime}\right|^{4}} d x \cdot d x^{\prime}\right.\right. \\
& \left.\left.-\frac{(a-x) \cdot d x^{\prime}}{|a-x|^{4}} \frac{\left(a-x^{\prime}\right) \cdot d x}{\left|a-x^{\prime}\right|^{4}}\right]\right) .
\end{aligned}
$$

Let us now specify to $n=4$. Computing the $\mathscr{P}$-ordered integrals in (4.12) we obtain for generic locations of 4 null cusps

$$
\mathscr{C}_{d i l}\left(W_{4}^{(r e g)}, a\right)=-\frac{\hat{c}_{d i l} \lambda}{2 \pi^{4}} \frac{\left|x^{(1)}-x^{(3)}\right|^{2}\left|x^{(2)}-x^{(4)}\right|^{2}}{\prod_{i=1}^{4}\left|a-x^{(i)}\right|^{2}} .
$$

\footnotetext{
${ }^{6} \mathrm{Up}$ to the scalar and the fermion equation of motion terms $\mathscr{O}_{\text {dil }}$ is thus given by the YM Lagrangian plus the Yukawa and the quartic scalar interaction terms.
} 
This agrees with the expected structure (2.13) of the correlator (for the dilaton $\Delta=4$ ) with the leading weak-coupling term in the function $F(\zeta)$ thus being simply a constant

$$
F(\zeta)=-\frac{\hat{c}_{d i l} \lambda}{2 \pi^{4}} .
$$

Note that the structure of (4.13) is exactly the same as the one appearing in the 1-loop correction to the 4-cusp Wilson loop $\left\langle W_{4}\right\rangle$ (given by a scalar box diagram). Indeed, integrating (4.13) over $a$ we get the integrated dilaton operator or gauge theory action insertion into the Wilson loop, which is proportional to derivative of $\left\langle W_{4}\right\rangle$ over gauge coupling $[9,11]$. This observation may allow one to extract higher order corrections to (4.13) by comparing to integrands of higher-order corrections to $\left\langle W_{4}\right\rangle$.

When computing the analogs of the integrals in (4.12) for $n>4$ we have two different types of contributions. The first one is when the two line integrals are taken along the same segment. Let us call this contribution $T_{i i}$ where the $i$-th segment is parametrized by (4.5). After some computation we obtain (up to the obvious factor $-\frac{\hat{c}_{d i l} \lambda}{\pi^{4}}$ )

$$
T_{i i}(a)=-\frac{1}{2} \frac{\left[\left(a-x^{(i)}\right) \cdot\left(x^{(i+1)}-x^{(i)}\right)\right]^{2}}{\left[\left(a-x^{(i)}\right) \cdot\left(a-x^{(i)}\right)\right)^{2}\left(\left(a-x^{(i)}\right) \cdot\left(2 x^{(i+1)}-a-x^{(i)}\right)\right]^{2}} .
$$

The other type of contribution appears when the two contractions are made in different segments. In this case we obtain

$$
\begin{aligned}
& T_{i j}=\frac{\left(a-x^{(i)}\right) \cdot\left(a-x^{(j)}\right)\left(x^{(i+1)}-x^{(i)}\right) \cdot\left(x^{(j+1)}-x^{(j)}\right)}{\left|a-x^{(i)}\right|^{2}\left|a-x^{(j)}\right|^{2}\left(a-x^{(i)}\right) \cdot\left(a+x^{(i)}-2 x^{(i+1)}\right)\left(a-x^{(j)}\right) \cdot\left(a+x^{(j)}-2 x^{(j+1)}\right)} \\
& -\frac{\left(a-x^{(i)}\right) \cdot\left(x^{(j+1)}-x^{(j)}\right)\left(a-x^{(j)}\right) \cdot\left(x^{(i+1)}-x^{(i)}\right)}{\left|a-x^{(i)}\right|^{2}\left|a-x^{(j)}\right|^{2}\left(a-x^{(i)}\right) \cdot\left(a+x^{(i)}-2 x^{(i+1)}\right)\left(a-x^{(j)}\right) \cdot\left(a+x^{(j)}-2 x^{(j+1)}\right)} .
\end{aligned}
$$

These expressions are completely general. Hence, the full answer (which is rather lengthy) will be the sum of such contributions.

Let us specify (4.15), (4.16) to the case of regular polygons with even $n$ sides with the cusps located at

$$
x^{(i)}=\left((-1)^{i} \frac{\sqrt{1-\cos \frac{2 \pi}{n}}}{1+\cos \frac{2 \pi}{n}}, \frac{\cos \left(\frac{\pi}{n}(2 i+1)\right)}{\cos \frac{\pi}{n}}, \frac{\sin \left(\frac{\pi}{n}(2 i+1)\right)}{\cos \frac{\pi}{n}}, 0\right) .
$$

The problem is purely combinatorial, but there does not seem to be a simple universal formula for generic $n$. It is relatively easy, however, to compute the OPE coefficient by placing the operator very far from the loop: taking $|a|$ large we obtain (cf. (2.4),(2.15))

$$
\mathscr{C}_{\text {dil }}\left(W_{n}^{(r e g)}, a\right)_{|a| \rightarrow \infty}=\frac{\mathrm{C}_{n}}{|a|^{8}}, \quad \mathrm{C}_{n}=-\frac{2 \hat{c}_{d i l} \lambda}{\pi^{4}} n^{2} \tan ^{2} \frac{\pi}{n} .
$$

For generic location of the dilaton operator one can check that the result is consistent with the general expectation (2.7). For instance, for $n=6$ and the case of a regular polygon the result depends on three conformal ratios (since the polygon is regular only three cross-ratios are independent) and we obtain

$$
F\left(\zeta_{1}, \zeta_{2}, \zeta_{3}\right)=-\frac{\hat{c}_{d i l} \lambda}{2 \pi^{4}} \frac{\zeta_{1} \zeta_{2} \zeta_{3}\left(\zeta_{3}-1\right)+\zeta_{3}^{2}-\zeta_{2}^{3}}{\left[\zeta_{1} \zeta_{2}^{2} \zeta_{3}^{2}\left(\zeta_{2}-\zeta_{3}\right)^{2}\left(\zeta_{1} \zeta_{3}\left(\zeta_{2}-1\right)-\zeta_{2}^{2}+\zeta_{3}\right)\right]^{1 / 3}}
$$


where the conformal ratios are defined by

$$
\begin{aligned}
& \zeta_{1}=\frac{\left|x^{(1)}-x^{(3)}\right|^{2}\left|a-x^{(5)}\right|^{2}}{\left|x^{(1)}-x^{(5)}\right|^{2}\left|a-x^{(3)}\right|^{2}}, \quad \zeta_{2}=\frac{\left|x^{(2)}-x^{(4)}\right|^{2}\left|a-x^{(6)}\right|^{2}}{\left|x^{(2)}-x^{(6)}\right|^{2}\left|a-x^{(4)}\right|^{2}}, \\
& \zeta_{3}=\frac{\left|x^{(1)}-x^{(4)}\right|^{2}\left|a-x^{(3)}\right|^{2}\left|a-x^{(6)}\right|^{2}}{\left|x^{(3)}-x^{(6)}\right|^{2}\left|a-x^{(1)}\right|^{2}\left|a-x^{(4)}\right|^{2}} .
\end{aligned}
$$

\section{Concluding remarks}

Here we considered, following [16], the correlator (2.1) of a null $n$-polygon Wilson loop with a local operator, such as the dilaton $\left(\mathscr{O}_{d i l} \sim \operatorname{tr} F_{m n}^{2}+\ldots\right)$ or a chiral primary operator. Based on symmetry considerations we determined its general form (2.7), expressing it in terms of a function $F$ of $3 n-11$ conformal ratios involving the position of the operator and the positions of the cusps. In the first non-trivial case of $n=4$ this function $F$ depends on just one conformal ratio $\zeta$ making the corresponding correlator (2.1),(2.13) one of the simplest non-trivial observables that one would like eventually to compute exactly for all values of the 't Hooft coupling $\lambda$. The value of $F$ determines, in particular, the corresponding OPE coefficient (2.16) in the expansion (2.3) of the Wilson loop in terms of local operators.

We have found the leading terms in $F$ both at strong coupling (using semiclassical string theory) and at weak coupling (using perturbative planar gauge theory). At leading order at strong coupling we find that $F \sim \sqrt{\lambda}$ and has non-trivial dependence on $\zeta$ (3.15) while at leading order in weak coupling $F \sim \lambda$ and is constant (4.14). In the case of more general dilaton operator with nonzero R-charge $j$ (with $\Delta=4+j$ ) the strong-coupling expression for $F$ is given by a hypergeometric function (3.24). Similar results were found in the case of the chiral primary operator (3.30),(3.32).

It would be important to compute subleading terms in the two respective expansions:

$$
\begin{aligned}
& F_{\lambda \gg 1}=\frac{1}{N}\left[\sqrt{\lambda} f_{0}(\zeta)+f_{1}(\zeta)+\frac{1}{\sqrt{\lambda}} f_{2}(\zeta)+\ldots\right], \\
& F_{\lambda \ll 1}=\frac{1}{N}\left[\lambda h_{0}+\lambda^{2} h_{1}(\zeta)+\lambda^{3} h_{2}(\zeta)+\ldots\right] .
\end{aligned}
$$

Another open problem is the extension to the case of the $n>4$ cusped Wilson loop.

Let us note that in the case of the dilaton operator integrating (2.1) over the point $a$ we get the insertion of the action and so the resulting correlator should be proportional to a derivative over $\lambda$ of the logarithm of the null-polygon Wilson loop. Thus, in particular, the knowledge of $\left\langle W_{n}\right\rangle$ at higher orders in $\lambda$ provides a constraint on integral of (2.1) at lower order order in $\lambda$; in general, this is not, however, enough to determine the functions $h_{n}(\zeta)$ in (5.2).

Part of the original motivation for the present work was to shed more light on the relation [15] between a correlator of null-separated local operators and the square of corresponding cusped Wilson loop. We conjectured a more general relation (1.7) connecting correlators with one extra operator at an arbitrary position to the correlator (2.1) we considered in this paper. It would be interesting to try to verify the relation (1.7) for $n=4$ at weak coupling.

There are several possible extensions of our present work. One may consider the case when the local operator $\mathscr{O}$ is not "light" at strong coupling but is allowed to carry a large charge (e.g., R-charge or angular momentum in $S^{5}$ so that $\Delta \sim \sqrt{\lambda}$ ). As in the circular loop case in [13], then the 
semiclassical surface will need to be modified to account for the presence of the sources provided by the vertex operator $\mathrm{V}$ in the string path integral (see also [14]).

One may consider also a correlator of a Wilson loop with several "light" $(\Delta \ll \sqrt{\lambda})$ operators. At leading order in strong-coupling expansion such a correlator should factorize like in the case of the correlators two "heavy" $(\Delta \sim \sqrt{\lambda})$ operators and several "light" ones $[22,26]$, i.e. $\left\langle W_{n} \mathscr{O}\left(a_{1}\right) \mathscr{O}\left(a_{2}\right)\right\rangle \sim\left\langle W_{n} \mathscr{O}\left(a_{1}\right)\right\rangle\left\langle W_{n} \mathscr{O}\left(a_{2}\right)\right\rangle$. This follows from the fact that for $\sqrt{\lambda} \gg 1$ these correlators are found, like in (3.3), by evaluating the corresponding vertex operators on the world surface ending on the null polygon that defines $W_{n}{ }^{7}$ The study of such more general correlators may be of interest in trying to understand better the relation [15] between the correlator of null-separated local operators and the square of corresponding cusped Wilson loop.

Finally, let us mention some recent related work that appeared in [36, 37, 38].

\section{Acknowledgments}

We would like to thank F. Alday and E. Buchbinder for a collaboration on ref. [16] on which this contribution is based. This work is partially supported by the ERC Advanced grant No.290456.

\section{References}

[1] N. Beisert et al., "Review of AdS/CFT Integrability: An Overview," arXiv:1012.3982.

D. Serban, "Integrability and the AdS/CFT correspondence," J. Phys. A 44, 124001 (2011) [arXiv:1003.4214].

[2] L. F. Alday and J. M. Maldacena, "Gluon scattering amplitudes at strong coupling," JHEP 0706, 064 (2007) [arXiv:0705.0303].

[3] G. P. Korchemsky, J. M. Drummond and E. Sokatchev, "Conformal properties of four-gluon planar amplitudes and Wilson loops," Nucl. Phys. B 795, 385 (2008) [arXiv:0707.0243].

[4] A. Brandhuber, P. Heslop and G. Travaglini, "MHV Amplitudes in N=4 Super Yang-Mills and Wilson Loops," Nucl. Phys. B 794 (2008) 231 [arXiv:0707.1153].

[5] L. F. Alday and R. Roiban, "Scattering Amplitudes, Wilson Loops and the String - Gauge Theory Correspondence," Phys. Rept. 468, 153 (2008) [arXiv:0807.1889]. J. M. Henn, "Duality between Wilson loops and gluon amplitudes," Fortsch. Phys. 57, 729 (2009) [arXiv:0903.0522].

[6] I. A. Korchemskaya and G. P. Korchemsky, "On lightlike Wilson loops," Phys. Lett. B 287, 169 (1992). A. Bassetto, I. A. Korchemskaya, G. P. Korchemsky and G. Nardelli, "Gauge invariance and anomalous dimensions of a light cone Wilson loop in lightlike axial gauge," Nucl. Phys. B 408, 62 (1993) [arXiv:hep-ph/9303314].

[7] M. Kruczenski, "A Note on twist two operators in N=4 SYM and Wilson loops in Minkowski signature," JHEP 0212, 024 (2002) [arXiv:hep-th/0210115].

[8] Y. Makeenko, "Light cone Wilson loops and the string / gauge correspondence," JHEP 0301, 007 (2003) [arXiv:hep-th/0210256].

\footnotetext{
${ }^{7}$ Contribution of a more singular term proportional to a power of $\left|a_{1}-a_{2}\right|^{-2}$ is suppressed at large $\lambda$. Note also that in general $\left\langle W_{n} \mathscr{O}\left(a_{1}\right) \mathscr{O}\left(a_{2}\right)\right\rangle$ should depend, say for $n=4$, on $3 n-11+4=5$ conformal ratios, this strong-coupling factorization implies that the leading term depends only on $2 \times(3 n-11)=2$ conformal ratios.
} 
[9] J. M. Drummond, J. Henn, G. P. Korchemsky and E. Sokatchev, "Conformal Ward identities for Wilson loops and a test of the duality with gluon amplitudes," Nucl. Phys. B 826, 337 (2010) [arXiv:0712.1223].

[10] A. M. Polyakov, “Gauge Fields as Rings of Glue,” Nucl. Phys. B 164, 171 (1980).

[11] G. P. Korchemsky and A. V. Radyushkin, "Loop space formalism and renormalization group for the infrared asymptotics of QCD”, Phys. Lett. B 171, 459 (1986). "Renormalization of the Wilson Loops Beyond the Leading Order," Nucl. Phys. B 283, 342 (1987). G. P. Korchemsky and G. Marchesini, "Structure function for large $\mathrm{x}$ and renormalization of Wilson loop," Nucl. Phys. B 406, 225 (1993) [arXiv:hep-ph/9210281].

[12] D. E. Berenstein, R. Corrado, W. Fischler and J. M. Maldacena, "The operator product expansion for Wilson loops and surfaces in the large N limit," Phys. Rev. D 59, 105023 (1999) [arXiv:hep-th/9809188].

[13] K. Zarembo, “Open string fluctuations in $A d S_{5} \times S^{5}$ and operators with large R charge," Phys. Rev. D 66, 105021 (2002) [arXiv:hep-th/0209095].

[14] L. F. Alday and A. A. Tseytlin, "On strong-coupling correlation functions of circular Wilson loops and local operators,” J. Phys. A A 44, 395401 (2011) [arXiv:1105.1537 [hep-th]].

[15] L. F. Alday, B. Eden, G. P. Korchemsky, J. Maldacena and E. Sokatchev, "From correlation functions to Wilson loops," arXiv:1007.3243.

[16] L. F. Alday, E. I. Buchbinder and A. A. Tseytlin, "Correlation function of null polygonal Wilson loops with local operators,” JHEP 1109, 034 (2011) [arXiv:1107.5702 [hep-th]].

[17] M. Kruczenski, R. Roiban, A. Tirziu and A. A. Tseytlin, "Strong-coupling expansion of cusp anomaly and gluon amplitudes from quantum open strings in $A d S_{5} \times S^{5}$," Nucl. Phys. B 791, 93 (2008) [arXiv:0707.4254].

[18] S. Ryang, "Conformal SO(2,4) Transformations of the One-Cusp Wilson Loop Surface," Phys. Lett. B 659, 894 (2008) [arXiv:0710.1673].

[19] S. S. Gubser, I. R. Klebanov and A. M. Polyakov, "A Semiclassical limit of the gauge string correspondence,” Nucl. Phys. B 636, 99 (2002) [arXiv:hep-th/0204051].

[20] S. Frolov and A. A. Tseytlin, "Semiclassical quantization of rotating superstring in AdS(5) x S5," JHEP 0206, 007 (2002) [arXiv:hep-th/0204226]. S. Frolov, A. Tirziu and A. A. Tseytlin, "Logarithmic corrections to higher twist scaling at strong coupling from AdS/CFT," Nucl. Phys. B 766, 232 (2007) [arXiv:hep-th/0611269].

[21] E. I. Buchbinder and A. A. Tseytlin, “On semiclassical approximation for correlators of closed string vertex operators in AdS/CFT,” JHEP 1008, 057 (2010) [arXiv:1005.4516].

[22] R. Roiban and A. A. Tseytlin, "On semiclassical computation of 3-point functions of closed string vertex operators in $A d S_{5} \times S^{5}$," Phys. Rev. D 82, 106011 (2010) [arXiv:1008.4921].

[23] K. Zarembo, "Holographic three-point functions of semiclassical states," JHEP 1009 (2010) 030 [arXiv:1008.1059].

[24] M. S. Costa, R. Monteiro, J. E. Santos and D. Zoakos, "On three-point correlation functions in the gauge/gravity duality,” JHEP 1011, 141 (2010) [arXiv:1008.1070].

[25] L. F. Alday and J. Maldacena, "Null polygonal Wilson loops and minimal surfaces in Anti-de-Sitter space," JHEP 0911, 082 (2009) [arXiv:0904.0663]. "Minimal surfaces in AdS and the eight-gluon scattering amplitude at strong coupling," arXiv:0903.4707 . 
[26] E. I. Buchbinder and A. A. Tseytlin, "Semiclassical four-point functions in $A d S_{5} \times S^{5}$," JHEP 1102, 072 (2011) [arXiv:1012.3740].

[27] A. M. Polyakov, “Gauge fields and space-time,” Int. J. Mod. Phys. A 17S1, 119 (2002) [arXiv: hep-th/0110196]. A. A. Tseytlin, "On semiclassical approximation and spinning string vertex operators in $A d S_{5} \times S^{5}$," Nucl. Phys. B 664, 247 (2003) [hep-th/0304139].

[28] L. F. Alday and J. M. Maldacena, “Comments on operators with large spin,” JHEP 0711, 019 (2007) [arXiv:0708.0672].

[29] E. I. Buchbinder, "Infrared Limit of Gluon Amplitudes at Strong Coupling," Phys. Lett. B 654, 46 (2007) [arXiv:0706.2015].

[30] E. I. Buchbinder, "Energy-Spin Trajectories in $A d S_{5} \times S^{5}$ from Semiclassical Vertex Operators," JHEP 1004, 107 (2010) [arXiv:1002.1716].

[31] L. F. Alday, "Review of AdS/CFT Integrability, Chapter V.3: Scattering Amplitudes at Strong Coupling," [arXiv:1012.4003].

[32] D. Gaiotto, G. W. Moore and A. Neitzke, "Wall-Crossing in Coupled 2d-4d Systems," arXiv:1103.2598 [hep-th].

[33] J. M. Maldacena, "Wilson loops in large N field theories,” Phys. Rev. Lett. 80, 4859 (1998) [hep-th/9803002]. S. J. Rey and J. T. Yee, "Macroscopic strings as heavy quarks in large N gauge theory and adS supergravity,” Eur. Phys. J. C 22, 379 (2001) [hep-th/9803001].

[34] L. Andrianopoli and S. Ferrara, “K-K excitations on AdS(5) x S5 as N=4 'primary' superfields," Phys. Lett. B 430, 248 (1998) [arXiv:hep-th/9803171]. S. Ferrara, M. A. Lledo and A. Zaffaroni, "Born-Infeld corrections to D3-brane action in $\operatorname{AdS}(5)$ x $\mathrm{S}(5)$ and $\mathrm{N}=4, \mathrm{~d}=4$ primary superfields," Phys. Rev. D 58, 105029 (1998) [arXiv:hep-th/9805082]. I. R. Klebanov, W. Taylor and M. Van Raamsdonk, "Absorption of dilaton partial waves by D3-branes," Nucl. Phys. B 560, 207 (1999) [arXiv:hep-th/9905174].

[35] H. Liu and A. A. Tseytlin, "Dilaton - fixed scalar correlators and $A d S_{5} \times S^{5}-\mathrm{SYM}$ correspondence," JHEP 9910, 003 (1999) [arXiv:hep-th/9906151].

[36] O. T. Engelund and R. Roiban, "On correlation functions of Wilson loops, local and non-local operators," arXiv:1110.0758 [hep-th].

[37] T. Adamo, "Correlation functions, null polygonal Wilson loops, and local operators," JHEP 1112, 006 (2011) [arXiv:1110.3925 [hep-th]].

[38] R. Hernandez, "Semiclassical correlation functions of Wilson loops and local vertex operators," arXiv:1202.4383 [hep-th]. 